\title{
Why Reprocess Seismic Data
}

Felipe Zumba Amorim, Gabriel de Almeida Araujo, Luiz Henrique Gomes Popoff, Pedro Henrique Cáceres Figueiredo, Wander Nogueira de Amorim

(ClmaGeo - Centro de Imageamento Geofísico)

\section{Copyright 2019, SBGf - Sociedade Brasileira de Geofísica}

This paper was prepared for presentation during the $16^{\text {th }}$ International Congress of the Brazilian Geophysical Society held in Rio de Janeiro, Brazil, 19-22 August 2019.

Contents of this paper were reviewed by the Technical Committee of the $16^{\text {th }}$ International Congress of the Brazilian Geophysical Society and do not necessarily represent any position of the SBGf, its officers or members. Electronic reproduction or storage of any part of this paper for commercial purposes without the written consent of the Brazilian Geophysical Society is prohibited.

\begin{abstract}
We present a discussion of the relevance of seismic data reprocessing whenever performing seismic interpretation of any nature. Seismic data from any database tends to have several stages of acquisition and / or processing, which impels them to a heterogeneity capable of hindering or even rendering it impossible to use as a whole. The arguments range from the quality of imaging to the mistakes made in storing the data.
\end{abstract}

\section{Introduction}

In order to carry out regional mapping, area evaluation or even exploration or exploitation well lease studies, the interpretation team of an oil company brings together all the available collection of surface or sub-surface data of the area of interest and carries out a study that allows to define if the existent data are sufficient and of quality that allow a reliable analysis for the proposed objectives.

Drilling for oil wells generally accounts for most of the costs of a given exploration campaign, so every effort should be made to give the project a good reliability in the expected results.

All bibliography, previous works, surface mappings, seismic maps, well data and their correlations, geochemical, gravimetric, magnetometric, or any other kind of surveys that permit to define geological characteristics of that area, previous results of success or failure are cataloged and analyzed and reinterpreted meticulously, in order to make the minimum risk possible for this new exploratory venture in that area.

All existing interpretations should be reviewed and, if necessary, redone, resulting in new maps, correlations and new perspectives for the area.

When the team considers that some data does not meet the needs, the possibilities of new surveys, reprocessing, interpretations or reanalysis are evaluated, in order to have a reliable and sufficient set of information for conclusions with sufficient technical background for the completion of the work.
For the case of indicating points for the location of wells, this study should indicate if there are viable prospects for the area and what would be the success rate of the leads, if this is the final conclusion of the work.

The reflection seismic, 2D or 3D, represents the most accurate tool for the structural definition and relations between layers of subsurface geology in sedimentary basins. This way geologists, geophysicists and reservoir engineers can have a high-resolution image of the layers and in many cases it is possible to reliably estimate the elastic properties of rocks by acoustic or elastic inversions of the data.

Typically interpretation teams work with well-defined project deadlines and costs, which can typically impact decision making.

New surveys of seismic reflection data should only be considered if existing data are insufficient due to scarcity or lack of quality to reach the goals with good reliability, since the cost and time involved in this step are high.

On the other hand, if the existing data density is sufficiently good, reprocessing of preexisting seismic reflection data may be a good alternative for increasing reliability of interpretation. In many cases the costs and deadlines involved for this task are low and you always get a good gain in the quality of the imaging with more current programs, besides being possible to homogenize the processing flow and suppress errors, which makes the dataset more easily interpretable.

\section{Discussion}

Several reasons may be raised for the need to reprocess geophysical data.

This discussion always comes to the fore when we are going to do some type of study in the areas where we want to evaluate the oil potential or indicate points for the lease of exploratory and / or exploratory wells.

- $\quad$ Are the existing data sufficient?

- Are existing data reliable?

- Is the database reliable?

- Is it possible to conduct further surveys in the areas of interest?

- Is there any homogeneity of acquisition and / or processing in the data?

- $\quad$ Does the need for reprocessing justify the cost? 
- Is there time for reprocessing?

- $\quad$ Are there companies with capacity and availability to carry out the service?

Thinking only of the seismic data of reflection, the answers to the above questions are not always simple or precise.

Depending on the objectives of the work, a higher or lower data density is required, for example, if the goal is to perform a regional mapping, a $2 \mathrm{D}$ seismic data set with sparse mesh may be sufficient, but if the objective is the detailing of Leads for bidding presentation of wells may require a mesh of $2 \mathrm{D}$ dense lines or even 3Ds and if the goal is to conduct production development studies, the data should be as dense as possible and processed with tools other than those in which only a study of layer geometry is being sought, often requiring acoustic or elastic inversions.

Considering that the database is sufficient, we analyze the reliability of the available data. In this case, a detailed analysis of both the individual data and the database as a whole is necessary, considering image quality, acquisition and / or process homogeneity, misties between lines, reliability in amplitude ratios, fault positioning, geodetic positioning of lines, datum and equations for the calculation of static corrections, polatiry of lines, among others. The database may be in-house or may be acquired from a larger database, in the case of Brazil, from the Banco de Dados de Exploração e Produção (BDEP) of the Agência Nacional do Petróleo, Gás Natural e Biocombustíveis (ANP)

In both cases, it is common to have a heterogeneous database, with several phases of surveys and processing. The seismic data acquired at different times may have a lot of variation in the acquisition parameters, caused by the technological advances of the equipment or the parameterization performed because of the objectives intended at that time. In both cases there may be great variation in the intrinsic quality of the different acquisitions, which can result in difficulties in using them in the same project.

In the case of processing at different times, the same variation in the quality of the results can be caused by the technological advances of the processing tools, either by new theories or by the improvement of the equipment used for the work, that allow greater precision in the applications.

Thus, the use of data with different processing and different times can cause a lot of difficulty and imprecision in the interpretation, and it is advisable whenever possible to process the entire data set with a single software and preferably with the same team, which would certainly add much value to the data set and would greatly reduce uncertainties and exploratory risks.

In the case of land data, one of the main problems with different processing is the accuracy of the static corrections, which starts with the methodology used until the equation for calculating the final corrections, which can vary greatly between different software.

Another serious problem may be the polarity of data came from different processing rows i with different processing center or people.
When using a database that groups surveys of different companies and times of acquisition or processing, a large number of problems can be found, ranging from those related to differences in image quality, to geodetic positioning of the data.

The following are some examples that try to justify the need to process the complete set of data in a given area.

\section{Geodetic positioning error}

Figure 1 shows a seismic line of the Solimões Basin with positioning error caused by the erroneous indication of the UTM Zone at the line header. Note that because of the Projection Zone error in the Header line, instead of 22S hey put 21S. There was a displacement of hundreds of kilometers.

\section{Imaging problem}

Figure 2 shows an example with evident gain in the quality of imaging achieved with the reprocessing of the data. Part $B$ of the figure clearly shows the gain in imaging quality obtained with the reprocessing of the seismic line, giving greater reliability in the interpretation.

\section{Velocity Analysis}

Stacking and migration velocities analysis is another key factor that always justifies the reprocessing of seismic data, since the interpretation of the seismic velocities is determinant in the quality of the image, besides that a poor estimation of the speeds can lead to a modification of the geology interpreted, even failing the composition of a basin, as suggested by Pereira et al., 2015.

\section{Static Correction Problem}

Figure 3 shows the result achieved with the application of more accurate methods of calculating static corrections. It can be observed that the undulations in the reflectors may be related to the inaccuracies in the calculation of the static corrections. There is also a great gain in quality in imaging, probably provided by the most current technology and best treatment of multiple reflections, which appear to be present in processing and attenuated in reprocessing.

\section{Data positioning error in the final datum}

Figure 4 shows a seismic line that was stored without applying the static to the final datum. Part $A$ of the figure shows the seismic data of the database, initially it was thought that the error was related to the calculation of the static corrections, however it was verified that there was not the complete application of the static corrections for the final datum, staying in the floating datum. The quality gain of the imaging is also significant with the reprocessing shown in part B of Figure, positioned in the floating datum for comparision.

Difference in the polarity of intersecting seismic lines 
Figure 5 shows the crossing of two 2D seismic lines stored with polarity error of one of them. The polarity error presented by the left line makes it appear to be a mis-tie problem, although the problem was caused by the wrong polarity for the storage. In land data it is very difficult to define polarity errors and this information must be precise and must be present in the seismic data header whenever.

\section{Error or lack of seismic line topography}

Figure 6 shows an example of a seismic line where the topography in the documentation was wrong. The figure shows the topographic profile of the seismic line coming from the documentation (BLUE) and the same profile taken from the satellite (RED). This type of error can be detected if there is a quality control where the topography of all lines is compared to satellite topography. Confirmed the error tries to get the correct data in the database to continue processing.

The examples presented are a small sample of what can be achieved with the reprocessing of seismic data and the importance of doing so in order to have a confidence in interpretation. Experience shows that whenever seismic data are reprocessed there is an expressive improvement in quality, often leading to changes in the geological design of a sedimentary basin.

In this way, whenever the geological and geophysical interpretation of a given area is to be conducted, it is recommended that the analysis of the existing data be performed in detail and the decision to reprocess or not be made, depending on the credibility that this set generates in the interpretation team.
All seismic data deserves to be processed. The gain obtained reprocessing seismic data depends on how old is the vintage processing, the governmental seismic database, the information saved in observer and processing reports and in the trace headers of seismic data. Depends also on technical capacity of the processing center that performs the reprocessing, represented in their processing workflow, and depends on the interaction between processing and interpretation parties and the quality control along the reprocessing stage. Besides, the improvement of final seismic section, the reprocessing allows to detect bad geological interpretations done on the old sections.

\section{Acknowledgments}

To the Central de Imageamento Geofísico Ltda. (Geophysical Imaging Center Ltda.) - ClmaGeo, for the availability of time and data for the accomplishment of the work. Companies that authorized the publication of seismic data images of their property. To the Brazilian Society of Geophysics - SBGf, for the presentation of the work at its 16th International Congress.

\section{Recommended Reading}

Brazilian Society of Geophysics - SBGf, 2012. Seismic Data Processing: advances in search of quality. Bulletin SGBf, 3, 2012.

Pereira, C., A. L. Farias, J. M. de Morais Neto, R. de Barros Gelli, L. G. Miranda, F. V. Capucci, and I. T. Junior, 2015, New processing and seismic interpretation for the northern sao francisco basin, brazil: Presented at the 14th International Congress of the Brazilian Geophysical Society \& EXPOGEF, Rio de Janeiro, Brazil, 3-6 August 2015, Brazilian Geophysical Society.

\section{Conclusions}
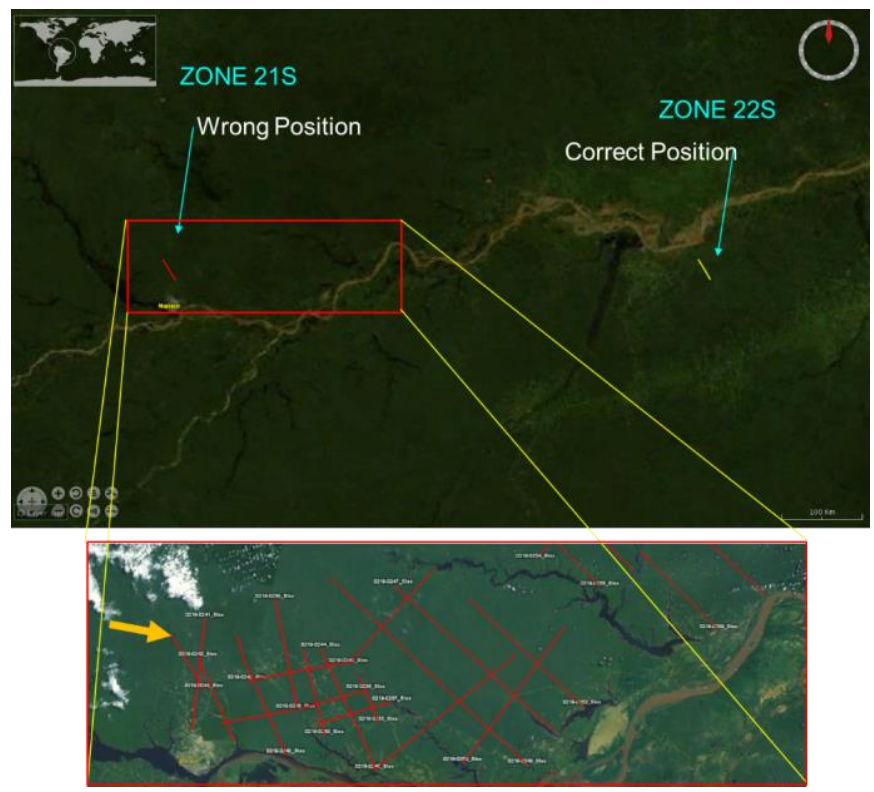

Figure 1 - Seismic line with positioning error (error in the UTM Zone). 

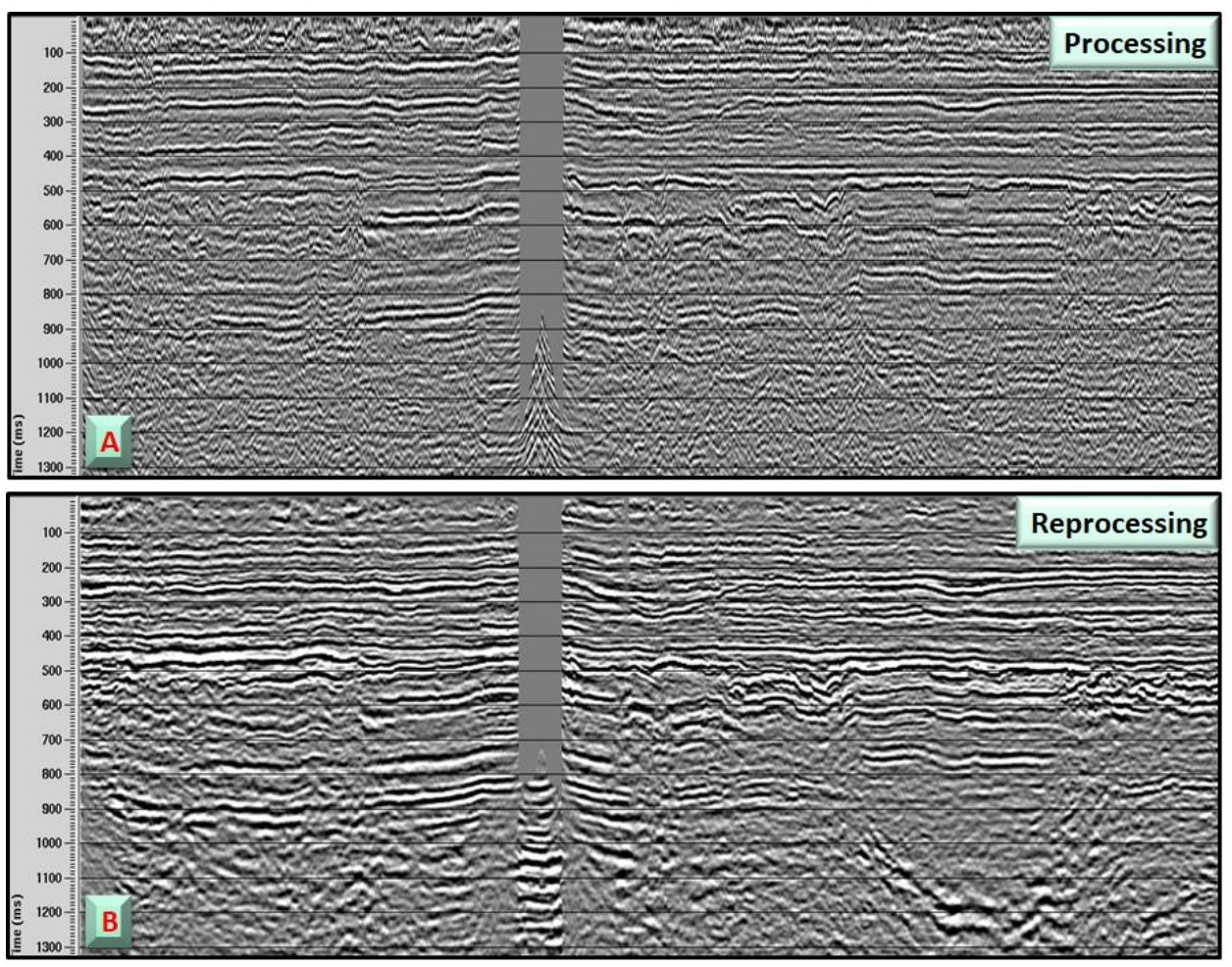

Figure 2 - Gain in imaging quality.
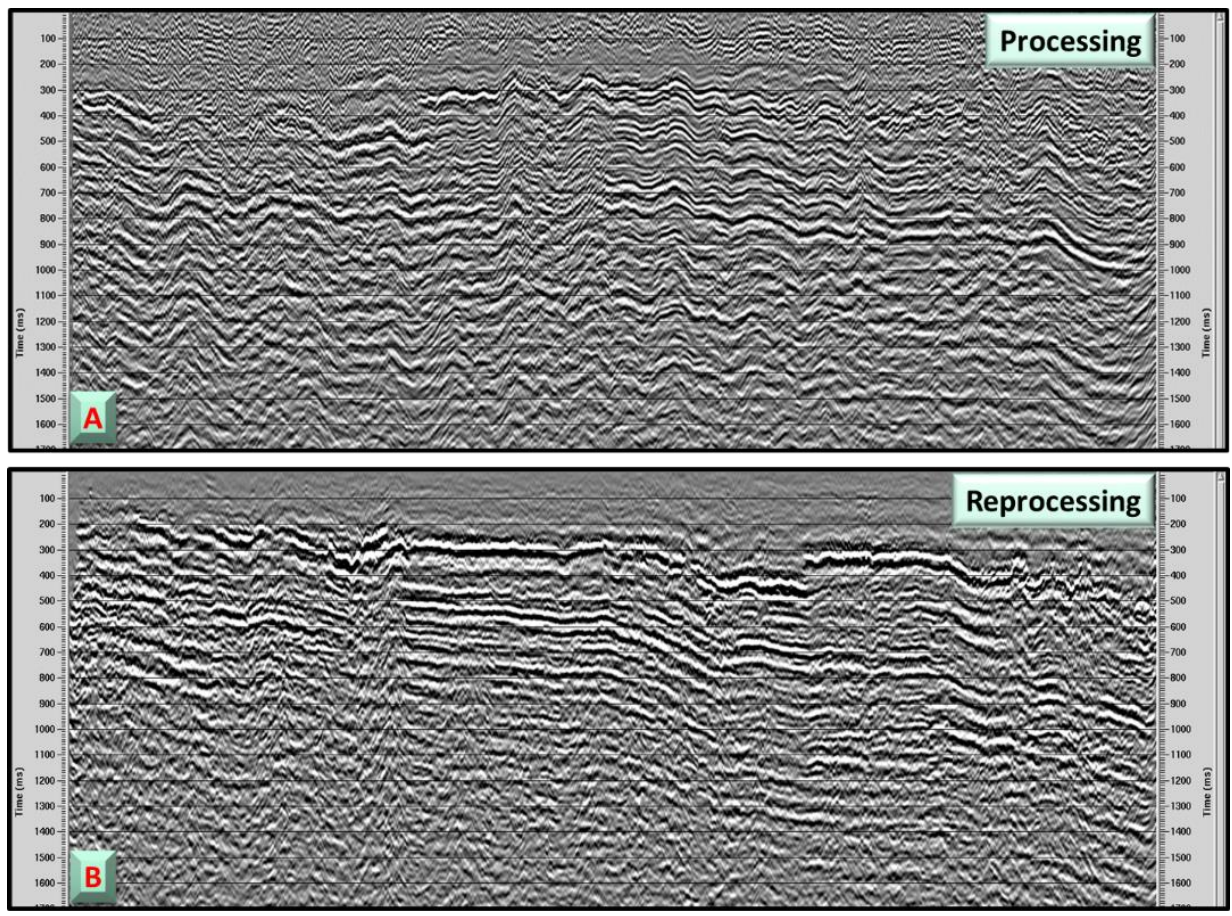

Figure 3 - Gain in quality due to static corrections with greater precision. 

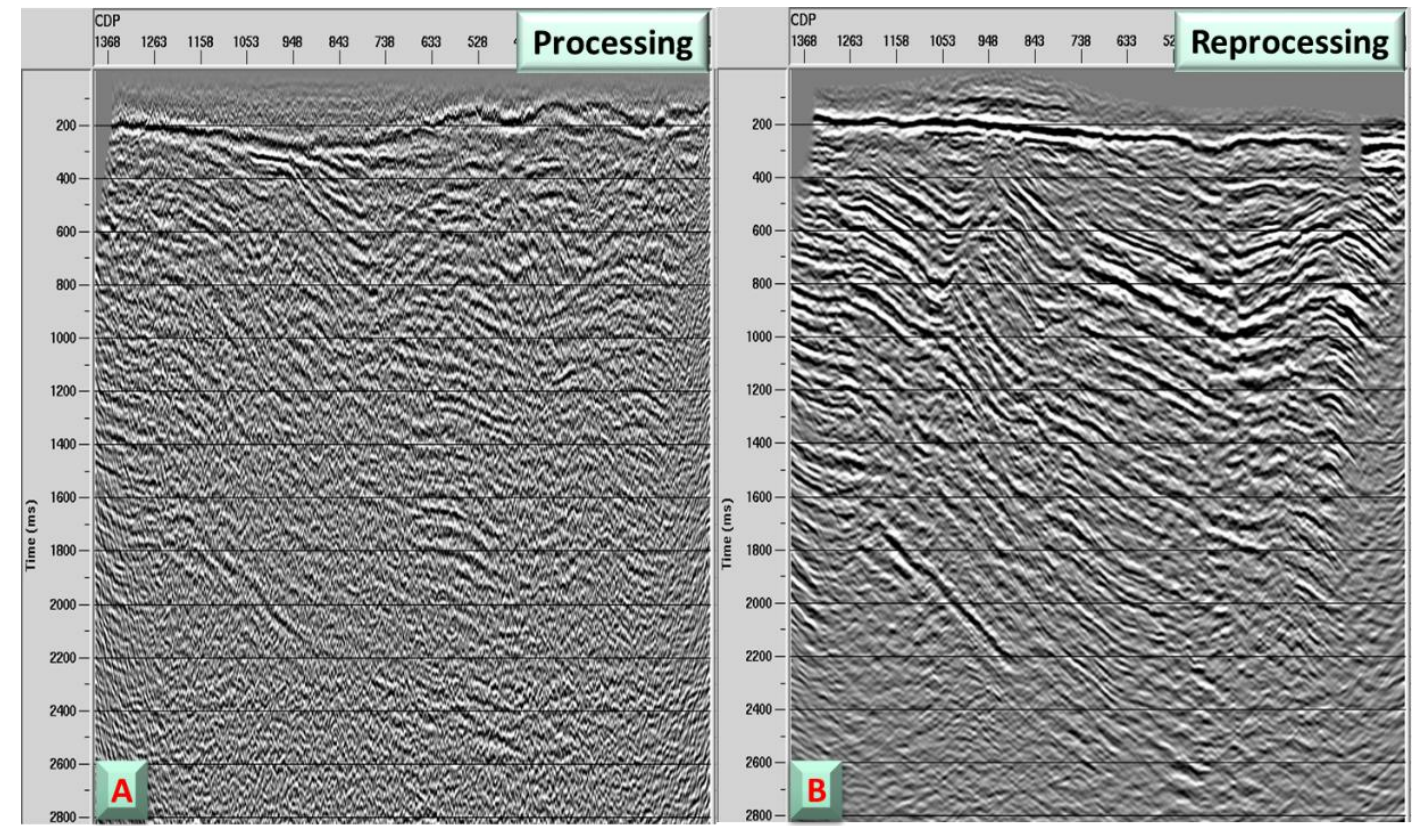

Figure 4 - Data positioning error in the Floating Datum.

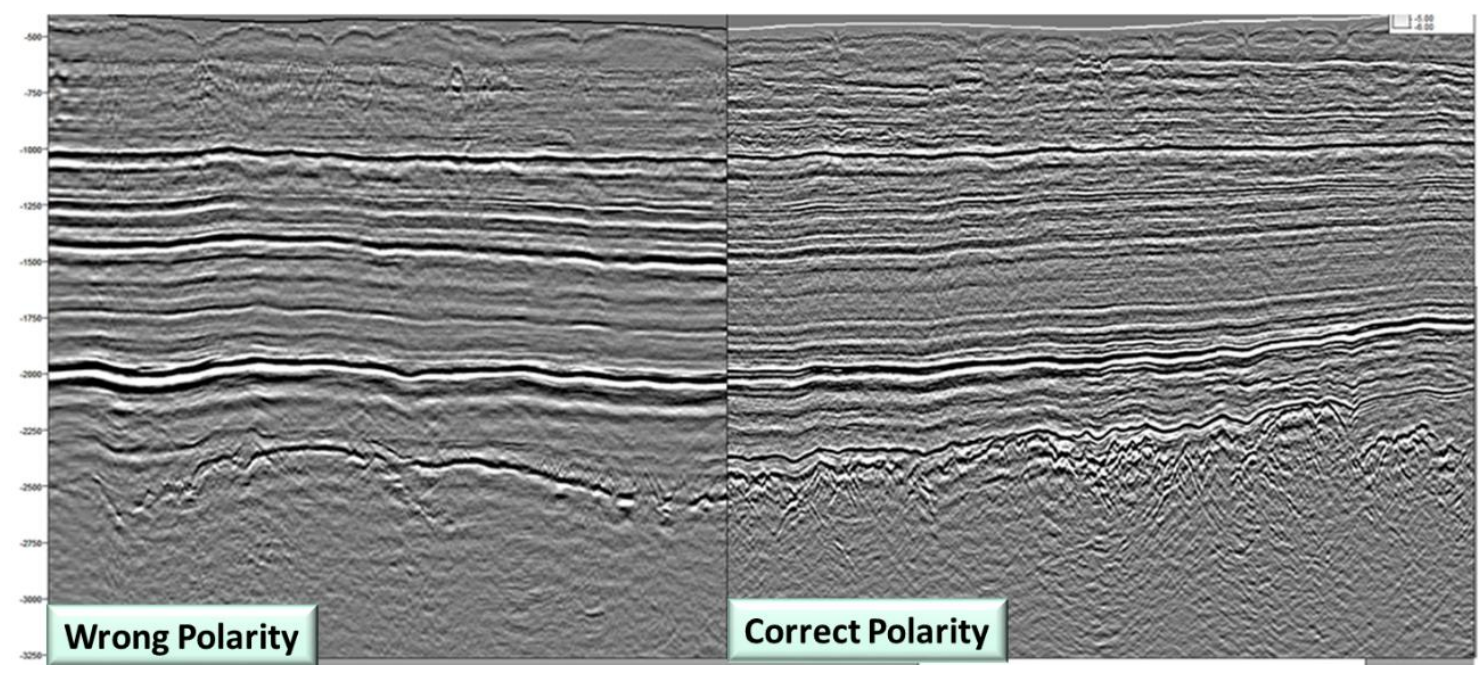

Figure 5 - crossing of seismic lines with polarity error in one of them.

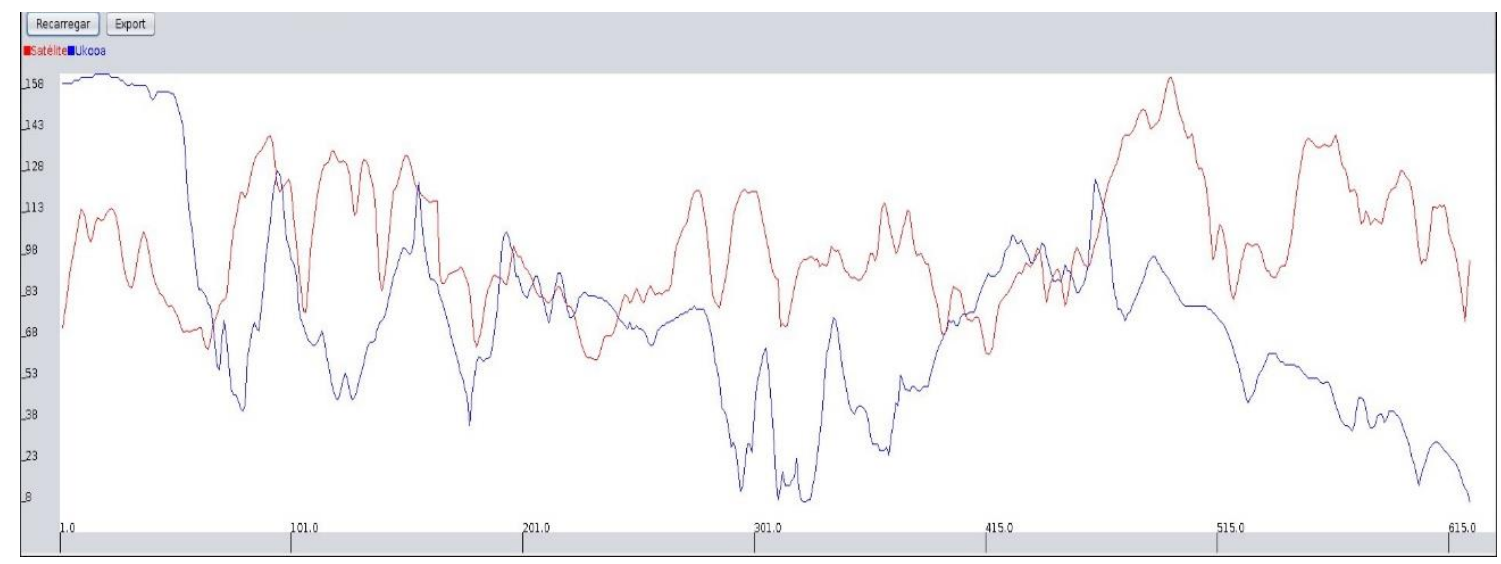

Figure 6 - Topography error of the seismic line. 